\title{
The Influence of Aerobic Exercise and Physical Education on the Physical Fitness of the Elderly
}

\author{
Yuzhong Chi and ZhiMing Xiao
}

Bao tou normal College of Inner Mongolia University of science and Technology

Keyword: Physical education; Aerobic exercise; Elderly people; Physical fitness and health

\begin{abstract}
Along with the growth of the age,children and parents communicate less and less, The physiological function of the elderly decreased in different degress, In order to slow down the descent rate,To improve their quality of life,we must actively and scientifically exercise.This paper expounds the influence of different types of aerobic exercise on the physical health of the elderly.
\end{abstract}

Through many aspects of scientific participation in aerobic exercise,the elderly to avoid sports hypoxia damage,prevent disease,Delaying senility and providing theoretical basis for health.

\section{有氧运动体育教育对老年人体质健康的影响}

\author{
池瑜忠，肖志民 \\ 内蒙古科技大学包头师范学院体育学院 014030
}

摘要: 随着年龄的增长, 老年人的各系统生理机能出现不同程度的下降。为了提高老年人体质健康, 延缓机能下降速度, 提高他们的生活质量，就必须积极科学的体育锻炼。本文介绍了不同类型有氧运动的体育教育对老年人体质健康的影响，分 析得出科学地参加有氧运动对老年人避免运动中缺氧损害、预防疾病、延续衰老和增进健康具有积极的作用。

关键词: 体育教育; 有氧运动; 老年人; 体质健康

\section{1 前言}

21 世纪是我国已进入老龄化社会, 随着老年人人数逐年增加, 已成为我们不得不重视的人群。根据老 年人生理解剖特点: 身体多项指标下降进而体质健康出现各种问题。如何改变或是延缓这样的发展速度, 已成为老年人必须面对的问题。有氧运动就是以增强体质, 预防疾病, 促进身体健康为目的的有效可靠方 法和手段。随着社会经济的不断发展, 我国老年人的健康认知也在提高。然而如何进行合理、科学、适宜 的体育锻炼, 始终是困扰这一人群的现实问题, 甚至有些老年人在这以问题上有很多的误区, 最终导致出 现运动性疾病和运动性损伤。最近新闻报道，欧洲国家德国是较早进入老龄化社会的国家之一。其老年人 的体育教育身体素质和体育健康居世界首位，国民平均寿命达八十一岁。而我国国民平均寿命七十六岁。 有人说这也许和种族有关。那么再看看东亚国家日本其国民平均寿命排在我们前面。他们早已在老年人体 质健康和身体素质方面进行了大量的研究探讨。形成了一整套较为完善的体育教育体系和实施方案。日本 有八十多岁登珠峰运动员, 当然这里不是提倡每个老年人都去训练, 登珠峰, 而是说明良好的身体素质, 我国幅员辽阔, 人口众多, 特别是广大的农村地区, 现在经济富裕了, 生活奔小康, 渴望健康, 希望从运 动中获取健康需求。为满足这种要求, 首先要从教育抓起, 充分利用各种渠道和方式加强科学锻炼的教育, 虽然我们在这方面做了一定的工作但还是不能满足需求。 


\section{2 有氧运动的教育}

有氧运动是美国健身专家肯尼斯・库伯斯首先提出，有氧运动即运动时能量供给是通过有氧代谢渠道 完成而非无氧酵解提供能量, 有氧运动对人体运动和健康是有益的。无氧酵解所产生的代谢中间产物乳酸 和酮体, 这些酸性物质对老年人的身体健康极为不利。这些物质的存在极易产生疲劳现象的产生。有氧运 动要求呼吸满足运动的需求, 强调呼吸与动作的协调, 对心肺功能的提高也有积极作用。

有氧运动其运动程度中低程度、有节奏、运动持续时间长的特点。每次持续的时间应控制在 $30-60 \mathrm{~min}$, 每周参加锻炼 3-5 次, 运动时对老年人的心率要求是: (200-年龄) 次/分, 通过这种训练, 老年人体各器 官系统机能得到提高, 预防甚至治疗老年人某些多发常见的疾病。也就是使老年人有亚健康状态, 疾病状 态转变为健康状态。有氧运动的项目包括, 健步走, 游泳, 有氧健身操, 健美广场舞以及一些民族体育等。 运动方法简单、易行，可以自检自控，安全有效。

\section{3 老年人的运动、心血管、呼吸和神经系统生理特点}

\section{1 运动系统的特点}

由于内分泌和代谢功能的改变，老年人的运动系统中骨骼出现萎缩和疏松，有机物含量减少，无机物 含量增多容易发生骨折, 随着肌纤维变细数目减少, 肌肉体积变小肌肉力量下降。老年人关节活动幅度减 小, 其柔韧和灵活性降低。肌肉收缩能力减弱、适应能力和抵抗力减弱。关节软骨附近会出现不同程度的 骨质增生、关节滑囊僵硬等原因, 造成老年性的退化性变化。

\section{2 心血管系统的特点}

老年人心血管系统主要在于心脏实质细胞减少, 以及供应心脏血管营养的冠状动脉出现不同程度斑块 状硬化, 导致心肌供血不足, 心肌收缩力下降, 由于低密度脂蛋白在血管儿壁的沉着使血管腔变窄, 血液 循环外周阻力加大，出现血压增高甚至高血压疾病。

\section{3 呼吸系统的特点}

老年人的呼吸机能明显减退，呼吸肌力量减退、肋软骨钙化、韧带弹性减弱使胸廓的活动度减小，使 肺脏的通气和换气功能降低。此外, 有的老年人胸廓脊柱还会出现各种变形, 更加加重了呼吸功能的衰竭。

\section{4 神经系统的特点}

老年人大脑逐渐萎缩、退化。大脑皮层表面面积和流经大脑血液均匀相应减少，大脑皮质神经活动过 程的灵敏性减弱, 现实生活中, 老年人的记忆能力, 注意力, 反应能力的下降且极易产生疲劳。疲劳消除 缓慢。这些都和其神经系统调节差, 对刺激不敏感有关。

\section{4 科学的、合理的、适宜的有氧运动体育教育能促进老年人体质健康发展}

随着我国老年大学的不断发展和壮大，老年人可通过体育教育更加准确地了解自身生理特点，根据实 际情况科学地进行体育锻炼。长期进行有氧运动达到提高心肺功能改善神经系统的兴奋性, 起到调节人的 心理和情绪的作用。通过老年人积极参与体育锻炼对身体各器官的良好影响, 可以有效地增进健康, 缓解 衰老过程, 起到延年益寿的良好作用。温克尔 (L. M. wanke1) 在他的有养运动流畅感和锻炼坚持性关系的 研究中认为: 一方面有氧运动使老年人生理健康也更容易坚持, 另一方面能使老年人获得心理健康, 出线 流畅感、幸福感、成就感。 


\section{1 步行运动与健康}

步行运动强调在一定的时间内达到一定的运动强度, 从而能到达提高心肺功能、调节免疫力功能。同 时它也是最简单的有氧运动项目, 步行运动特点表现为简单轻快、缓和、对老年人锻炼身体是一个很好的 选择。健步走的过程中由于足底和地面的撞击, 可起到刺激人体足底反射区。对内脏器官起到一定的效果。

研究表面适当强度的健步走是老年人最理想的运动方式之一，使老年人上下肢、肌肉与身体其他部位 协调配合。步行运动是以腹式呼吸为主, 腹式呼吸增加了膈肌的收缩和舒张, 促进呼吸系统功能, 良好的 步态对老年人纠正不良的姿势起到关键作用, 避免了因不良姿势造成的疾病潜在风险。

\section{2 太极拳运动与健康}

中华武术博大精深, 渊远流长。太极拳是中华武术养生精活所在, 它被作为老年人最理想的锻炼项目, 把太极拳运动当作锻炼方式时, 对养生和保健有着良好的效应, 他既可以有效地调动和发挥自身潜力, 增 强体。又能防病、祛病。

从现代医学角度看太极拳运动对提高老年人心肺功能有积极影响, 其特点: 凝神运气, 以静止动, 以 不变应万变, 呼吸自然、均匀、速度缓慢的思想理念。促进血液循环, 使人体更能充分适应自然环境, 做 到人天合一。太极拳的练习反而增加了老年人单脚站立的时间, 提高了下肢肌肉力量, 不仅提高人体肌肉 力量的均衡性, 还对其灵活性有很多的帮助。

从查阅文献资料中可以看出，太极拳运动作为民族传统养生经典部分传承至今，体现了其强大的生命 力, 对中华民族的健身, 养生, 保健做出了巨大的贡献。

\section{3 氧健身操运动与健康}

有氧健身操和健身广场舞深受老年朋友的喜爱和追捧，不仅提高了老年人身体健康水平，而且促进了 他们良好心理状态、社会适应能力, 以及良好的人际关系。有氧健身操动作比较简单, 强度和难度相对较 低, 而且练习时间可以根据个体情况而变化, 是一项适合老年人健身的运动。

有氧健身操对于老年人各器官系统机能提高有积极的影响。可提高老年人的新陈代谢, 提高各器官系 统的机能，降低老年人身体患病率，增强老年人身免疫力。

从查阅文献资料中知道, 有氧健身操运动被应用到阿尔茨海默病患者的辅助治疗上, 能提高人的认知 转换功能。

\section{4 游泳运动与健康}

游泳作为一项全身性参与运动项目，依靠自然界水对人体产生一定的浮力的特征再加上人体在水中协 调性运动技巧, 来满足运动需要的体验。游泳运动也是一项水中协调呼吸的运动。游泳运动对呼吸系统、 疾病患者是最理想的体育运动项目。它可以解除呼吸道平滑肌的痉挛, 改善呼吸器官的通气量, 达到提高 呼吸系统的目的。

老年人长期进行力所能及的游泳锻炼, 能有效地提高心肺功能, 增加骨密度, 减少骨质疏松症的风险。 同时还能使肌肉匀称伸缩, 使身体变得强健、灵活。游泳运动还可以益于人们养成良好的姿态, 矫正身体, 减少关节疼痛, 预防疾病, 促进康复。由于游泳平躺的姿势除了水对机体的按摩作用外, 由于水的浮力作 用, 减轻了关节由于体重而造成的负担, 特别是肥胖患者避免长期运动对膝关节的损伤, 同时运动中增加 了回心血量, 对身体形成良性循环。心脏每搏输出量的增加, 有利于心功能的提高。游泳运动是一项全身 
参与的运动, 与其他运动相比, 游泳时可以动员更多的肌肉群参与运动, 有利于全身肌力发展的均衡性和 协调性。长期进行游泳能增强机体抵御寒冷，适应环境的能力，可以预防感冒等。

从查阅文献资料中可以看出, 游泳运动可以作为运动处方中较为理想的运动项目。更适用于一些不适 合直立锻炼的老年人如过度肥胖症、膝关节退行性改变等患者, 如果采用其他的运动项目, 由于重力的作 用，下肢负担过重，容易导致受伤。

有氧运动是适合老年人健身的方式，合理营养结合长期的周期性有氧运动，可起到延缓衰老，益增天 年的功效。

\section{5 老年人有氧运动中的几个运动人体科学问题分析}

\section{1 老年人 21 世纪最合理的生活方式}

积极参加有氧运动且成为日常生活的一部分内容是本世纪老年人最合理的生活方式, 老年人不宜参加 速度型项目和力量型项目，运动负荷的大小是以心律为指标其适宜规范为 200-年龄/分

\section{2 老年人进行体育锻炼时，应学会如何选择有氧运动项目}

老年人和中青年人的显著区别特点在于体制差异较大，所以锻炼前的健康检查显得特别重要，尤其关 注心血管系统的情况, 比如脉搏和血压等, 这些都是老年人运动前选择运动项目的前提条件, 绝不可大意 轻心。老年人从事体育锻炼时, 必须根据自身情况, 量力而行。锻炼时要循序渐进, 持之以恒, 运动负荷 要从小到大逐渐增加, 增加的速度不宜过快。在自我监督方面, 老年人在参加有氧运动时要及时了解运动 中心率和血压的变化尤其更加注意晨脉和晨压的变化, 以便随时调整锻炼计划。活动中适当的安排短暂的 休息, 进行全身性的锻炼, 避免某器官运动负荷过重。同时注意运动性疲劳的产生, 做到劳逸结合及时消 除疲劳, 如有季节气候环境条件不适宜进行锻炼就暂停锻炼, 如果身体出现不适, 也同样注意休息和治疗。

\section{6 结论与建议}

\section{1 结论}

\section{1.1 有氧运动对老年人运动系统的影响}

有氧运动对老年人骨骼、软骨、肌肉、关节维持正常功能有积极作用，如促进肌肉代谢，提高肌肉耐 力和肌肉力量: 维持正常的钻磷代谢, 防止出现骨骼中的钻流失, 使骨密度增强, 骨的弹性和㓞性和骨的 成分含量相关，提高骨骼的新陈代使有机物含量所占比例增多，或延缓骨骼中有机物含量成分逐年减少的 趋势。对预防骨质疏松症有一定的疗效。对老年人来说, 也许有氧运动的最大价值是保持活力, 延缓衰老, 提高活动能力, 时老年人动作保持一定的幅度和协调性。

\section{1.2 有氧运动对老年人体循环和肺循环的影响}

心脏作为人体的最重要的器官之一, 它是建立人体血液循环的动力来源, 人体所需的各种物质和能量 就是依靠循环到达靶细胞和靶器官老年人进行有氧运动时, 能使机体循环血液中心分配。老年人长期参加 有氧运动不仅对提高身体各器官系统的功能, 同时对心脏本身的供给也相应得到改善。从而减缓心血管系 统机能随年龄的增加而下降的趋势。

\section{1 .3 有氧运动对老年人呼吸系统的影响}

有氧运动的呼吸过程中，能使呼吸和运动节奏更趋合理协调充分利腹式呼吸方式的特点，使更多呼吸 
肌参与工作, 增强呼吸肌力, 增加肺活量, 更多的排除体内残留二氧化碳气体, 吸取更多的新鲜空气。参 加有氧运动 1 年以上的老年人, 其肺活量、呼吸差、气体交换频率等指标均有显著增加, 起到促进细胞代 谢，延缓衰老。此外，长期参加室外体育锻炼可以预防老年人慢性支气管炎和肺部其他疾病。

\section{1.4 有氧运动对老年人神经系统的影响}

老年人长期参加有氧运动锻炼所产生的良好效应, 最主要的集中体现在心血管系统机能的改善, 随着 心血管系统机能的改善, 分布在脑组织的血管的其能相应发生良好改变脑组织的供血供养提高, 对预防老 年痴呆, 增强神经系统对人体的调节能力有着重要的作用。老年人长期有氧运动还能消除疲劳和神经紧张, 提高对外界环境的适应能力、保持精力旺盛、改善睡眠，防治老年性疾病。

\section{2 建议}

在全面建设实现小康社会, 建设具有中国特色社会主义的新时代当下, 我们要积极贯彻执行国家倡导 并逐步落实形成比较完善的现代国民教育体系，科技和文化创新体系，全民健身和医疗卫生体系，提高国 民 “健康素质”、“思想道德素质”、“科学文化素质”。加大宣传普及老年人科学地有氧健身运动的健康知 识, 提高老年人对有氧运动的认识, 组织老年人积极参加健身活动, 让老年人认识到只有进行长期的科学 有效地有氧运动，才是老年人本世纪最健康的生活方式。让老年人了解到有氧运动给身体带来的益处。根 据《中华人民共和国老年人权益保障法》的相关内容的规定，应该建立健全养老服务体系，加强社会组织 间交流合作。更为有效地促进老年人体质健康。

建议老年人的生活, 除了需要注意营养和平衡膳食以外，老年人参加有氧运动时，应该根据自身的情 况选择适宜的锻炼内容和方法, 参加运动时要量力而行, 每周锻炼 3-5 次, 每次锻炼时间持续 40min 以上 能达到显著的效果。有氧运动时, 要注意微汗即可, 不要大汗淋漓。此外, 产生的运动疲劳应该能在第二 天消除。

\section{参考文献:}

[1] 唐可欣，王金光，王风斌．步行运动对老年人呼吸循环系统功能的影响 [J]．中国临床康复， 2013.

[2] 孙校, 王否, 张秀英等. 太极拳对认知功能及精神健康影响的研究进展 [J]。中国老年学杂志, 2012，36 (17): 3844-3847.

[3] 间严. 太极拳运动对老年人心肺功能的影响研究 [J]. 辽宁师范大学学报 (自然科学版)，2013. 36 (1)：124-127

[4] 孙闽君，李爱民，周君华，林东梅. 有氧健身操对老年人生理机能影响的调查[J]. 现代预防医学. 2009

[5] 李亚楠. 有氧健身操对老年人心血管系统的影响 [J]．武汉体育学院学报，2009（39）

[6]刘崇，阎芬，曹冰等. 运动延缓老年人体质下降的研究 $[J]$. 中国康复医学杂志，2009（7）

\section{References}

[1] K.X.Tang, J.G.Wang and F.B.Wang: The Effect of Walking Exercise on the Function of the Respiratory and Circulatory System of Old People[J], Chinese Journal of Tissue Engineering Research, 2013.(In Chinese)

[2] J.Sun, L.Wang, X.Y.Zhang, etc: Research Progress on the Influence of Tai Chi on Cognitive Function and Mental Health[J], Chinese Journal of Gerontology, 2012, 36(17): 3844-3847.(In Chinese)

[3] Y.Yan: The Effect of Tai Chi Exercise on the Heart-Lung Function of the Aged People[J], Journal of Liaoning Normal University(Natural Science), 2013.36(1): 124-127.(In Chinese)

[4] J.Sun, A.M.Li, J.H.Zhou and D.M.Lin: An Investigation of the Effects of Aerobic Exercise on the Physiological Function of the Aged People[J], Modern Preventive Medicine.2009.(In Chinese) 
[5] .N.Li: Effects of Aerobic Exercise on the Cardiovascular System of the Aged People[J], Journal of Wuhan Institute of Physical Education, 2009(39).(In Chinese)

[6] .Liu, F.Yan, B.Cao, etc: Study on delaying physical decline of elderly people with exercise[J], Chinese Journal of Rehabilitation Medicine, 2009(7).(In Chinese) 